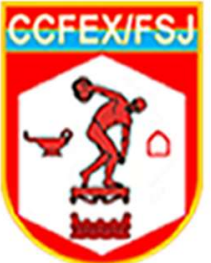

\title{
Revista de Educação Física

Historiografia

Historiography

\section{0, um problema de saúde em escala global: uma historiografia sobre a pandemia de CoViD-19 e aspectos relacionados à prática de atividade física}

\section{0, a Global Health Problem: A Historiography on the CoViD-19 Pandemic and Physical Activity Related Issues}

\author{
Lilian Martins ${ }^{\S 1} \mathrm{PhD}$; Renato Soeiro ${ }^{1} \mathrm{MSc}$
}

Recebido em: 10 de dezembro de 2020. Aceito em: 18 de dezembro de 2020.

Publicado online em: 22 de dezembro de 2020.

DOI: $10.37310 /$ ref.v89i3.2703

\section{Resumo}

Introdução: No início de janeiro de 2020, surgiram notícias difusas a respeito de uma nova doença, que logo se transformou em pandemia.

Objetivo: Examinar o desenrolar histórico desse agravo à saúde e identificar possíveis efeitos sobre a prática de atividade física.

Métodos: Foi feita busca nos termos "CoViD-19 or SARS-Cov2 or coronavirus" "WHO" "2020" compuseram o estudo artigos científicos, recomendações da Organização Mundial da Saúde (OMS) e notícias de jornais.

Resultados e Discussão: Os primeiros meses do ano configuraram-se em um período de grande apreensão, face ao enfrentamento da nova, e potencialmente mortal, doença. Com o desenvolvimento do conhecimento clínico e experimental, os mecanismos da doença foram sendo desvendados e as formas de tratar/prevenir a CoViD-19 foram sendo estabelecidas. Recomendações da OMS quanto ao uso de máscaras de proteção durante a prática de atividade física foram identificadas.

Conclusão: As consequências da pandemia atingiram todos os setores da vida humana, afetando a produtividade. É provável que a prevalência de sedentarismo tenha aumentado em todas as partes do globo, porém, os impactos sobre a prática de atividade física, cujos benefícios à saúde são bem estabelecidos na literatura, ainda não

\begin{tabular}{|l|}
\hline Pontos Chave \\
- Janeiro de 2020: uma \\
pandemia de um vírus \\
desconhecido, chamado "novo \\
coronavírus" atinge todo o \\
globo. \\
- O vírus teve origem na China \\
no segundo semestre de 2019, \\
assim a doença foi chamada de \\
CoviD-19. \\
- Todos os setores da vida \\
humana foram afetados pela \\
pandemia e os impactos sobre a \\
prática de atividade física ainda \\
são desconhecidos. \\
\hline
\end{tabular}
foram examinados.

Palavras-chave: exercício, pandemia, CoViD-19, determinantes sociais da saúde.

\begin{abstract}
Introduction: In early January 2020, diffuse news emerged about a new disease, which soon turned into a pandemic.

Objective: To examine the historical development of this health problem and to identify the possible effects on the practice of physical activity.

Methods: A search was made under the terms "CoViD-19 or SARS-Cov2 or coronavirus" and "WHO" and "2020" on Google Web and Scholar and PubMed. In this research was included: scientific articles published, recommendations from the World Health Organization (WHO) and newspaper articles.
\end{abstract}

$\S$ Autor correspondente: Lilian Martins - e-mail: lilitina@gmail.com

Afiliações: ${ }^{1}$ nnstuto de Pesquisa da Capacitação Física do Exército (IPCFEx), Rio de Janeiro - RJ, Brasil. 
Results and Discussion: Results and Discussion: The first months of the year were characterized by a period of great apprehension, given the confrontation of the new, and potentially deadly, disease. With the development of clinical and experimental knowledge, the mechanisms of the disease were being unraveled and the ways to treat/prevent CoViD-19 were being established. WHO recommendations regarding the use of protective masks during physical activity were identified.

Conclusion: Conclusion: The consequences of the pandemic affected all sectors of human life, affecting productivity. It is likely that the prevalence of sedentary lifestyle has increased in all parts of the globe, however, the impacts on the practice of physical activity, whose health benefits are well established in the literature, have not yet been examined.

Keywords: exercise, pandemic, CoViD-19, social determinants of health.

\section{Key Points}

- January 2020: a pandemic of an unknown virus, called "new coronavirus", hits the whole globe.

- The virus originated in China in the second half of 2019, so the disease was called CoviD-19. - All sectors of human life were affected by the pandemic and the impacts on the practice of physical activity are still unknown.

\section{0, um problema de saúde em escala global: uma historiografia sobre a pandemia de CoViD-19 e aspectos relacionados à prática de atividade física}

\section{Introdução}

O ano de 2020 foi um marco na história da humanidade. A partir do início do mês de janeiro, foram trazidas notícias difusas a respeito de uma nova doença, cujos primeiros casos foram relatados em Wuhan, na província de Hubei, na China(1). Em meio a um cenário de muitas dúvidas, enquanto o número de mortes crescia assustadoramente ao redor de todo o mundo - como se apresenta mais adiante no presente estudo, médicos, cientistas, governantes e populações buscavam por informações para se proteger da peste mortal que se acercava. A doença, que logo de início foi declarada como decorrente de um novo coronavírus, foi denominada CoViD-19, que é a abreviatura para coronavirus disease of 2019.

O objetivo do presente estudo foi examinar o desenrolar histórico desse agravo à saúde que afetou todas as vidas no planeta e, consequentemente, a produtividade em diversas áreas da vida humana e procurar identificar os possíveis efeitos sobre a prática de atividade física. Trata-se da primeira parte do trabalho que contextualiza o tema, que seguir-se-á de uma edição focalizando o que a ciência exibe quanto aos reflexos da pandemia'(2) relacionados a essa prática.

\section{Métodos}

Foi feita uma busca nos termos "CoViD-19 or SARS-Cov2 or coronavirus" e and "WHO" and "2020" no Google Web e Scholar e no PubMed. Integraram a presente pesquisa, além de artigos científicos publicados em periódicos científicos, recomendações da Organização Mundial da Saúde (OMS) e artigos de jornais de notícias.

\section{Resultados e Discussão}

\section{Cronologia}

30 de dezembro de 2019 - O jovem médico chinês Li Weliang tentou alertar seus colegas a respeito do novo coronavírus, que seria perigoso, foi advertido e preso pela polícia chinesa por espalhar inverdades(3).

Janeiro de 2020 - Enquanto pouco foi divulgado pela China a respeito do vírus quanto contágio e formas de propagação, foi à OMS que todos os países recorreram em busca de informações e recomendações para proteger e, quem sabe, curar suas populações. 
Entretanto, naquele momento, o assunto era bastante obscuro.

23 de janeiro de 2020 - A OMS afirmou que a transmissão de humano para humano não estava clara "the extent of human-to-human transmission is still not clear"(4).

24 de janeiro de 2020 - Um artigo publicado na Lancet, um dos periódicos médicos de maior prestígio, afirmou que se tratava de um surto de pneumonia de causa desconhecida e que, seria plausível que o contágio se desse por via aérea (airborne $)^{2}(5,6)$. Destaca-se que a literatura mostra registros desde 2002 de infecções respiratórias causadas por coronavírus, que levam à síndrome respiratória aguda severa (Severe Acute Respiratory Syndrome Coronavirus: SARS), havendo ocorrido pandemia em 2002-2003 (SARS-CoV) e epidemia em 2012 (Middle East respiratory syndrome coronavirus: MERS-CoV)(7).

Ao final do mês de janeiro, a doença já havia se espalhado pelo globo e o número de mortes, ocorridas, em curtíssimo espaço de tempo, foi extremamente alarmante: entre os dias 22 e 31 de janeiro (dez dias), esse número saltou de 17 para 259(8). Apesar disso, em 30 de janeiro, a OMS não recomendou qualquer restrição de viagens e, além disso, afirmou que quaisquer medidas tomadas, por qualquer país, em relação a viagens deveriam obrigatoriamente ser comunicadas à OMS, advertindo-os contra ações que promovessem estigmatização e/ou discriminação, invocando os princípios do artigo $3^{\circ}$ de suas Regulações Internacionais de Saúde de 2005 (International Health Regulations: IHR, 2005):

"The Committee does not recommend any travel or trade restriction based on the current information available.

Countries must inform WHO about travel measures taken, as required by the IHR. Countries are cautioned against actions that promote stigma or discrimination, in line with the principles of Article 3 of the IHR."

O que pode ter dificultado, ainda no início do problema, que barreiras fossem estabelecidas pelos países, que impedissem o aumento do contágio.

\footnotetext{
${ }^{2} \mathrm{Na}$ literatura em saúde, o termo airborne que especifica um tipo de contágio em que o agente patogênico permanece
}

Posteriormente, a literatura mostrou que a transmissão do coronavírus 2 seria por via aérea apenas a curta distância, devido ao seu alto peso molecular $(9,10)$ e que o contato próximo, ocorreria sobretudo a menos de 2 metros havendo fala muito alta, tosse ou espirro, sendo as formas mais comuns de contágio, destacando que a facilidade de propagação aumenta consideravelmente quando tais situações acontecem em ambiente fechado(5,9-15).

04 de fevereiro de 2020 - no Brasil, o Governo Federal decretou, preliminarmente, estado de emergência preparando ações para gerenciar no país a crise em saúde que se aproximava(16). Não obstante, o Carnaval no Brasil correu com normalidade, sobretudo nas principais cidades do país: São Paulo e Rio de Janeiro, que contam sempre com grande afluência de estrangeiros $(17,18)$.

20 de fevereiro de 2020 - Um estudo demonstrou que o vírus se propagava de humano para humano(19).

26 de fevereiro de 2020 - Foi registrado o primeiro caso confirmado de CoViD-19 no Brasil(20).

27 de fevereiro de 2020 - A OMS manifestou-se no tema turismo, ressaltando apenas que restrições de viagem extrapolativas poderiam causar interferência desnecessária no tráfego internacional, incluindo repercussões negativas no setor de turismo: "Travel restrictions going beyond these may cause unnecessary interference with international traffic, including negative repercussions on the tourism sector"'(21). Nesse dia, o registro de mortes por CoViD-19 ao redor do mundo era de 2.858 .

Março de 2020 - No mês de março, vários acontecimentos importantes tiveram lugar, configurando-se em período crítico na busca de ações para tratamento e prevenção da nova doença.

$1^{\circ}$ de março de 2020 - Um grupo de cientistas franceses, sob orientação do eminente médico pesquisador Dr. Didier Raoult, realizou um estudo piloto observacional, com um tamanho amostral de 80 pacientes com média de idade de 52 anos,

flutuando no ar de modo que, em ambiente fechado, a probabilidade de contágio aumenta muito(5). 
variando entre 18 e 88 anos, que apresentavam sintomas moderados de CoViD-19 utilizando hidroxicloroquina no tratamento. Os resultados mostraram que o uso de hidroxicloroquina, em associação com azitromicina, diminuiu a carga viral nasofaríngea em $83 \%$ ao sétimo dia e a $93 \%$ no oitavo dia(22). Resultados semelhantes in vitro foram publicados por Liu et al.(23), precisamente dia 18 de março.

07 de março de 2020 - A OMS destacou o registro de 100.000 casos de CoViD-19 no mundo e alerta que a propagação do vírus pode ser significativamente retardada ou mesmo revertida através da implementação de contenção robusta e atividades de controle, citando como exemplo a China e outros. E recomenda esforços para conter o vírus(24). Não obstante, os dados da China, fossem bastante distintos das demais curvas epidemiológicas de outros países, como se pode observar na Figura 1(25).

15 de março de 2020 - Foi publicada uma projeção estatística por um grupo de pesquisadores do Imperial College of London
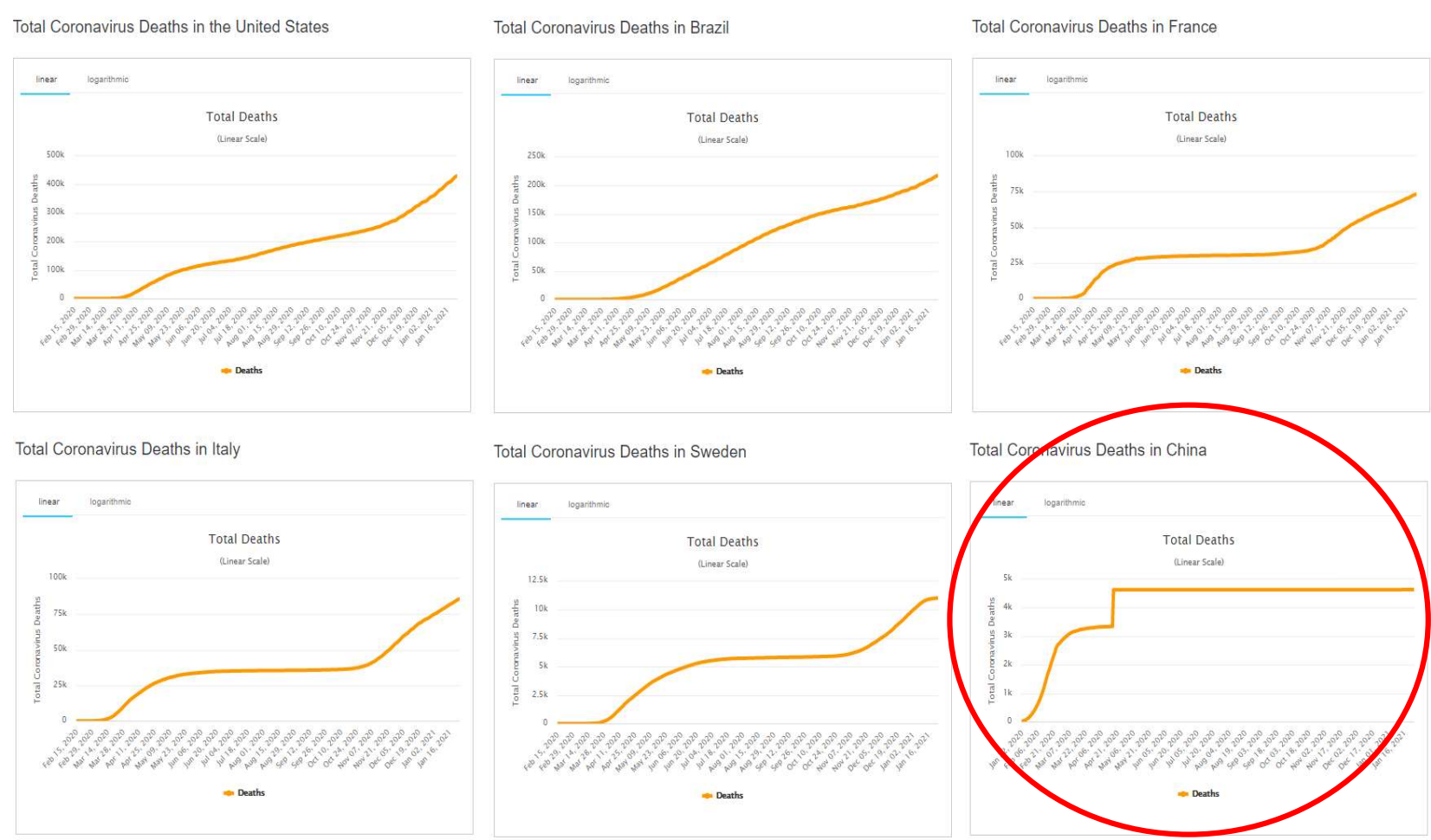

Figura 1 - Curva epidemiológica de mortalidade por CoViD-19 na Suécia (Sweden), Estados Unidos (United States), Reino Unido (United Kingdom), Brasil (Brazil), China, Itália (Italy), Israel e Espanha (Spain). Fonte: Worldometers(25).

cujos números para a previsão de mortes por CoViD-19 eram estarrecedores: morreriam 500 mil pessoas no Reino Unido e mais de 2.200 milhões nos Estados Unidos da América(26). E recomendaram que a principal estratégia prolítica para combater a pandemia seria o chamado lockdown, cujo termo a Agência Brasil(27) explica:

"No Brasil, o termo é previsto na diretriz até o momento vigente do Ministério da Saúde (MS). O Boletim Epidemiológico $n^{o}$ 8 , publicado em 9 de abril, define o lockdown como uma das medidas de distanciamento social. O bloqueio total (como o termo foi traduzido) consiste em cercar um determinado perímetro (estado, cidade ou região), interrompendo toda atividade por um breve periodo de tempo.

De acordo com a pasta, esse modelo tem como vantagem ser "eficaz para redução da curva de casos e dar tempo para reorganização do sistema em situação de aceleração descontrolada de casos e óbitos". A desvantagem é o alto custo econômico. 
Além dele, são modalidades menos rígidas o distanciamento seletivo $e$ o ampliado.

O primeiro seria aquele focado apenas em pessoas acima de 60 anos ou com doenças crônicas, os grupos de risco, além daquelas que apresentam sintomas da doença. Também conhecido como "isolamento vertical", teria como finalidade viabilizar o retorno às atividades laborais, já que não impossibilita a circulação de pessoas em estado aparentemente saudável ou que já passaram pela doença e são consideradas imunizadas.

Já o ampliado, que vem sendo adotado na maior parte dos estados e cidades, prevê que todos os setores fiquem em casa, à exceção dos essenciais, desde que nesses seja garantida higienização e evitada a aglomeração. De acordo com o Ministério, o objeto é "reduzir a velocidade de propagação, visando ganhar tempo para equipar os serviços com os condicionantes mínimos de funcionamento: leitos, respiradores, EPI [equipamento de proteção individual], testes laboratoriais e recursos humanos."

Nesse contexto de muita obscuridade, muitos países e muitos estados e municípios brasileiros aderiram ao lockdown recomendado pelo Imperial College(26) e pela OMS(4). Essa medida visava diminuir a velocidade do contágio para que os sistemas de saúde se preparassem para a grande quantidade de pacientes que surgiriam. A recomendação da OMS(4) para tais restrições era a de que fossem temporárias e por curto período de tempo (15 dias).

Como os governantes deixaram de observar a recomendação de curto prazo de tempo para restrições sociais, o problema que era de saúde, ganhou contornos políticos, uma vez que a ação de confinamento populacional geral, traz trágicas consequências para a economia, acarretando aumento da miséria extrema e mortes por fome(28), além de acarretar diversos outros problemas de saúde física(29) e mental(30-32).

Ainda no mês de março, a Câmara Internacional do Comércio (International Chamber of Commerce: ICC) reuniu-se com a OMS para coordenarem esforços a fim de juntos combaterem a pandemia por meio de assegurar informações recentes e confiáveis para orientar a comunidade comercial global(33). De acordo com a OMS:

'A pandemia de COVID-19 é uma emergência global de saúde na sociedade que requer ação imediata, e efetiva, por parte de governos, individuos e empresas. Todas as empresas têm um papel fundamental a desempenhar na minimização da probabilidade de transmissão e impacto na sociedade. Uma ação precoce, ousada e eficaz reduzirá os riscos de curto prazo para os funcionários e os custos de longo prazo para as empresas e a economia."

17 de março de 2020 - foi registrada, no Brasil, a primeira morte por CoViD-19.

20 de março de $\mathbf{2 0 2 0}$ - O número de mortes por CoViD-19 no mundo era de 11.610. No Brasil, o número de mortes saltou de 1 para 330, em apenas três dias. Nessa data, o Governo Federal brasileiro decretou estado de calamidade pública(34), quando, em diversos estados e cidades, começou o lockdown extendido.

30 de março de 2020 - A OMS já alertava para que precauções fossem tomadas para que a economia nos países não fosse desati$\operatorname{vada}(35)$ :

"Milhões de pessoas em todo o mundo dependem do comércio internacional para sua segurança alimentar e subsistência. À medida que os paises adotam medidas com o objetivo de deter a pandemia de COVID-19, deve-se tomar cuidado para minimizar os impactos potenciais sobre o abastecimento de alimentos ou consequências indesejadas no comércio global e na segurança alimentar."

Essas recomendações foram e continuaram sendo ignoradas pela grande imprensa e por vários governantes no Brasil e no mundo.

08 de abril de 2020 - As manifestações da OMS foram relacionadas ao problema de programa de prevenção da poliomielite, que poderia ficar prejudicada pela pandemia(36) e foi ressaltado o problema do aumento acentuado de violência contra a criança, durante $\mathrm{o}$ confinamento relacionado à pandemia(37). Ainda em abril, teve início a busca por vacinas(38).

No Brasil, a coordenação central do gerenciamento da pandemia foi, em grande medida, retirada do Governo Federal pela 
suprema corte(39). Assim, Estados e Municípios geriram e gerem, até a presente data, a crise da pandemia. No dia seguinte, o Ministro da Saúde, que divergia do Presidente quanto ao isolamento social (lockdown extendido) foi demitido(40).

A essa altura, a literatura quanto ao tratamento precoce utilizando-se a hidroxicloroquina / cloroquina - cujo primeiro estudo relacionado à CoViD-19, foi publicado em $1^{\circ}$ de março(22), exibia diversos estudos conduzidos em humanos, indicando que, associada à azitromicina, baixava consideravelmente a carga viral do paciente com sintomas moderados $(22,41,42)$. Adicionalmente, começam a surgir evidências de outro medicamento apontado como potente redutor da carga viral do novo coronavírus: a ivermectina(43). Além disso, as vitaminas D e Zinco foram apontadas como devendo estar em níveis adequados para favorecer a recuperação da saúde do indivíduo, possuindo papel importantíssimo no processo(44).
No Brasil, foram sendo desenvolvidos novos protocolos de tratamento precoce para o tratamento da CoViD-19 - isto é, na fase ambulatorial - antes de atingir o estágio de necessidade de internação hospitalar, dentre os quais podem ser citados: o estado do Piauí(45), o município de Porto Feliz-SP(46) e o protocolo do Ministério da Saúde do mês de maio(47). Um dos primeiros protocolos a ser aplicados foi o elaborado por médicos (clínicos e pesquisadores) e certificado pelo governo do Piauí(45).

20 de maio de 2020 - O Ministério da Saúde publicou novas orientações para o enfrentamento precoce da doença(47).

Destaca-se que um município, de Porto Feliz (população: 53.402 pessoas(48), no interior do estado de São Paulo, que elaborou, aplicou e aplica o protocolo de tratamento precoce, desde o dia 05 de abril(46) e, em 07 de dezembro de 2020, o boletim epidemiológico da cidade foi apresentado com os seguintes números: 2.521 casos, 2.478 curados, tendo

\section{BOLETIM EPIDEMIOLÓGICO}

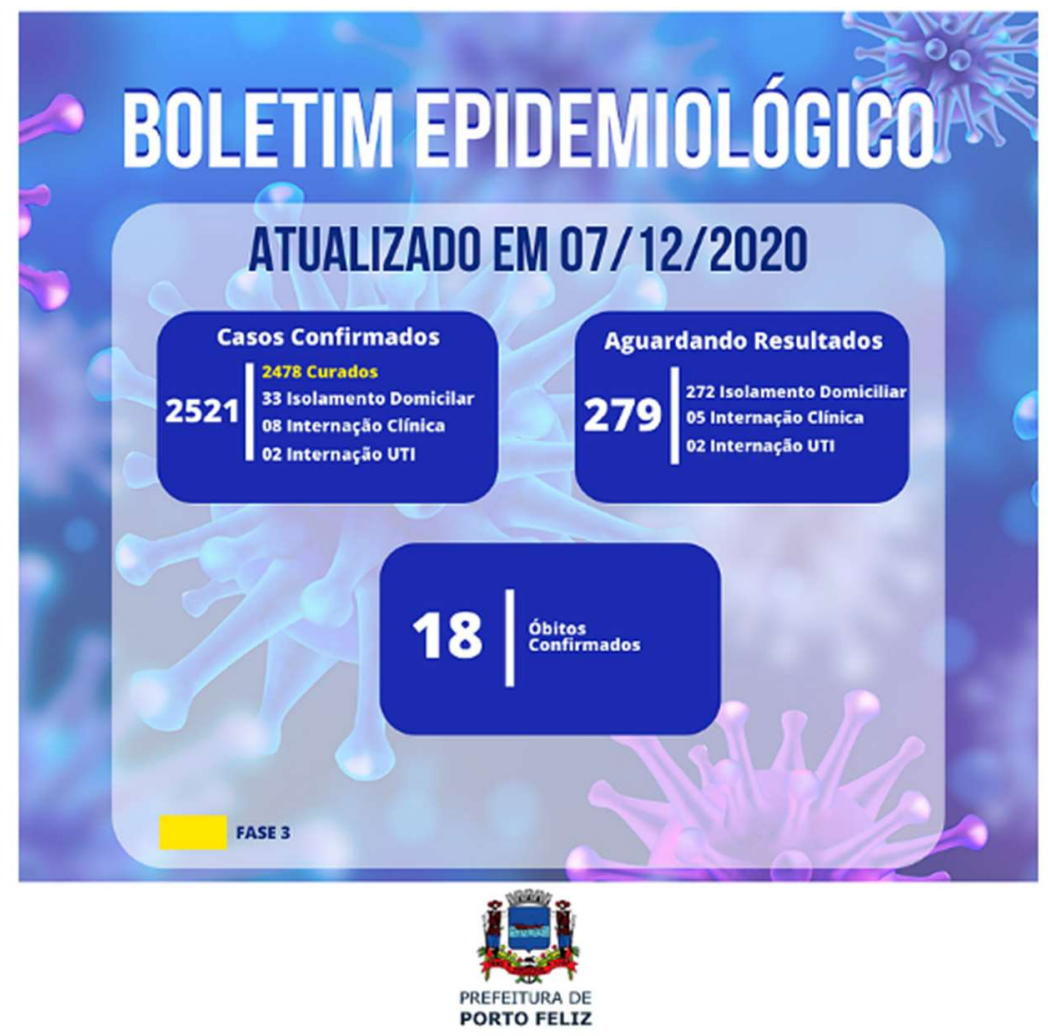

Figura 2 - Boletim epidemiológico de Porto Feliz - SP, 07 de dezembro de 2020. Fonte: Jornal Aliança. Porto Feliz(46) 
ocorrido 18 óbitos (Figura 2), exibindo uma taxa de mortalidade ${ }^{3}$ de 33,70 por 100.000 habitantes e letalidade ${ }^{4}$ de 0,01 . Na data de hoje, essas taxas, no Brasil, são: mortalidade ${ }^{3}$ de 83,96 por 100.000 habitantes e letalidade de 2,66E-02. A letalidade ${ }^{4}$ do novo coronavírus no mundo, na data em que se produz este artigo, é de 0,02, segundo dados do Worldometers $\AA(25)$.

31 de maio de 2020 - No dia seguinte ao estabelecimento do protocolo para 0 tratamento precoce da CoViD-19 do Ministério da Saúde, o estado do Piauí oficializou seu protocolo(45) que, segundo as pesquisas clínicas e estudos científicos, delineou as fases da doença, possibilitando a compreensão dos processos e os tratamentos específicos para cada uma delas.

\section{Um ano de muitas incertezas}

E desde o início do ano de 2020, o ser humano passou a conviver com mais esse agravo à saúde que, desconhecido, poderia ser mortal para determinadas pessoas. Nesse contexto, apesar dos avanços no conhecimento da doença(45), das recomendações da OMS(35) para a retomada das atividades e da declaração do Governo Federal de que atividades essenciais deveriam voltar a funcionar, desde antes de maio de 2020(50), a retomada das atividades em diversos estados e cidades, até hoje (meados de dezembro de 2020), encontram-se restritas e/ou proibidas em diversas regiões do país.

Tais restrições afetaram todas as áreas da vida humana nas sociedades ao redor do mundo, assim como no Brasil. Afetando a economia, aumentando o desemprego. Nesse cenário, há uma grande preocupação com as populações vulneráveis à pobreza que podem sofrer um impacto de grande número de mortes por fome, de acordo com o alerta da Organização das Nações Unidas (ONU), publicado ainda em meados de abril(28,51).

Além disso, houve um grande aumento em problemas de saúde mental. A literatura mostra que houve incremento na carga global de estresse, ansiedade, sintomas depressivos, insônia, negação, raiva e medo(52-54).

${ }^{3}$ Taxa de mortalidade segundo causa: é o número de óbitos por determinada causa divido pela população multiplicado por 100.000 (49).
Efeitos da restrição de circulação de pessoas sobre a saúde humana

Diminuição dos níveis de vitamina D

A restrição de circulação em lugares públicos afeta a imunologia do indivíduo sob vários aspectos. Primeiro, a elevada quantidade de tempo de permanência em ambiente fechado aumenta o risco para doenças respiratórias(11-15,55). Segundo, impossibilita que os indivíduos possam exporse ao banho de sol, origem a principal fonte de produção de vitamina $\mathrm{D}$ endógena, que é sintetizada na pele, principalmente após a exposição a UVB (raios ultravioleta B), enquanto apenas uma pequena parte dessa produção deriva-se de fontes dietéticas(56,57), sendo uma substância essencial à prontidão do sistema imunológico(58).

Outro fator que dificultou o manejo da doença foi, notadamente, a desinformação presente nos veículos de comunicação. Um exemplo que pode ser citado é que, apesar da literatura ser farta e consistente quanto aos benefícios da vitamina $D$ ao sistema imunológico - uma busca simples no PubMed (uma das principais bases científicas médicas) com os termos " $D$ vitamine" and. "immune", exibe 641 estudos de revisão, prévios à pandemia, com datas entre 1987 e 2019, foi veiculado na mídia que a vitamina $D$ não prevenia contra a CoViD-19(59-61). Tais notícias veiculadas em 2020, estavam na contramão do que a literatura científica exibia: que níveis baixos de vitamina $\mathrm{D}$ associam-se ao adoecimento por influenza, síndrome de imunodeficiência humana adquirida (SIDA ou Adquired Immunodeficiency Syndrome: AIDS)(62). O papel fundamental dessa vitamina foi, aos poucos, sendo estabelecido em informação à população em geral. Sobretudo quando um estudo epidemiológico do tipo caso-controle retrospectivo, conduzido na Espanha, que investigou a associação de nível de vitamina D com CoViD-19 identificou diferença significativa entre os pacientes internados $(n=216)$ e os controles $(n=197)$, sendo que os pacientes apresentavam níveis mais baixos de vitamina $\mathrm{D}$ em comparação

${ }^{4}$ Letalidade: é o número de óbitos divido pelo número de $\operatorname{casos}(49)$. 
com os controles(63), resultados que se apresentam em linha com outros estudos(56$58,62,64)$.

\section{Agravos à saúde mental}

Outro problema relacionado ao lockdown é que o estresse, o confinamento e o isolamento social causam problemas em saúde mental $(\mathbf{5 4 , 6 5 )}$. Os efeitos da pandemia foram que houve aumento na carga global de estresse, ansiedade, sintomas depressivos, insônia, negação, raiva e medo(52,54,65) e há a preocupação de que a próxima pandemia seja em saúde mental(66). Tais agravos em saúde mental, por sua vez, associam-se à imunodepressão(67,68) e associam-se a baixos níveis de atividade física(69-71).

Não obstante, até hoje, em diversos estados e municípios, alguns gestores continuam a impor o lockdown à sociedade.

\section{O uso de máscaras}

Além das restrições de circulação de pessoas, foram impostas regras e obrigatoriedades quanto ao uso de máscaras de proteção. As orientações mais recentes da OMS(72) quanto ao uso de máscaras, publicadas em $1^{\circ}$ de dezembro de 2020, apresentam-se a seguir.

\section{Em ambiente interno, a máscara de}

\section{proteção deve ser usada:}

- Quando há pouca ventilação: abertura limitada ou nenhuma abertura de janelas e portas para ventilação natural; o sistema de ventilação não está funcionando corretamente ou não apresenta manutenção em dia ou não pode ser avaliado;

- Em ambientes internos públicos com ventilação adequada se o distanciamento físico de pelo menos um metro não puder ser mantido;

- Em ambientes domésticos internos quando há um visitante que não é um membro da família e a ventilação é conhecida como ruim, com abertura limitada de janelas e portas para ventilação natural, ou o sistema de ventilação não pode ser avaliado ou não está funcionando corretamente; $\mathrm{e}$
- Em ambientes domésticos internos que tenham ventilação adequada se o distanciamento físico de pelo menos um metro não puder ser mantido.

\section{Em ambiente externo:}

- A recomendação é a de que pessoas com sintomas de quaisquer doenças respiratórias, em público devem usar máscaras médicas de proteção;

- Pessoas saudáveis deverão usar máscaras em público quando a distância física de pelo menos um metro não for possível de ser mantida;

- Pessoas com risco elevado para complicações sérias, no caso de contágio de CoViD-19 “(indivíduos $\geq 60$ anos e aqueles com condições subjacentes, como: doença cardiovascular ou diabetes mellitus, doença pulmonar crônica, câncer, doença cerebrovascular ou imunossupressão) devem usar máscaras médicas em qualquer local onde a distância fisica não puder ser mantida".

\section{O uso de máscaras durante a prática de atividade física}

A literatura quanto ao uso de máscaras de proteção durante a prática de atividade física é escassa. Até o momento não existem estudos que respondam, de modo conclusivo quanto a relação entre utilização de máscara e desempenho físico. Porém, a máscara cria uma barreira física entre a via respiratória e o ar ambiente, o que dificulta a mobilização de ar gerando um desconforto respiratório, podendo ocorrer uma hiperventilação compensatória, com consequentes aumentos da frequência cardíaca ou outros efeitos sobre a função cardíaca(73). Tais alterações, proporcionais à intensidade da atividade e ao nível de condicionamento físico, impactam na percepção do esforço. Um estudo demonstrou impactos desse uso sobre parâmetros fisiológicos cardiopulmonares em pessoas saudáveis, sendo que houve redução significativa na ventilação pulmonar de 131 1/min para 114 1/min(73) e redução de 37\% na 
troca respiratória com o uso de máscara de proteção(74). Ainda em pessoas saudáveis, o uso de máscaras modificou significativamente a dispneia ${ }^{5}$, sem influenciar a distância caminhada, e os resultados foram considerados clinicamente relevantes(76). Outro estudo registrou prejuízos, também em pessoas com doenças pulmonares(77).

Segundo a Sociedade Brasileira de Medicina do Esporte (SBME), a máscara de proteção pode trazer certo desconforto respiratório e relativa perda de rendimento, mas afirma que não há evidências de que seu uso coloca em risco a saúde do usuário que deseja praticar exercícios físicos(78). .

Segundo a OMS, as recomendações quanto ao uso de máscaras de proteção durante a prática de atividade física é a seguinte:

- Não se deve usar máscaras durante atividades físicas de intensidade vigorosa, mantendo o distanciamento físico de pelo menos um metro e garantindo uma boa ventilação no ambiente durante os exercícios.

- Se a atividade ocorrer em ambientes fechados, a ventilação adequada deve ser garantida em todos os momentos por meio da ventilação natural ou de um sistema de ventilação funcionando adequadamente ou mantido;

- Atenção especial deve ser dada à limpeza e desinfecção do ambiente, especialmente superfícies de alto contato. E acrescenta que, se todas as medidas anteriores não puderem ser garantidas, deve-se considerar $\mathrm{o}$ fechamento temporário das instalações de ginástica internas (condomínios) ou públicas (academias, clubes).

${ }^{5}$ Dispneia: falta de ar(75).

6 Atividade física: qualquer movimento corporal produzido por contração do músculo esquelético que aumente o gasto de energia acima do nível basal(80) e pode ser classificada em três tipos: $A$ atividade física ocupacional é a que se dá em razão de atividades ocupacionais. Por exemplo, subir e descer escadas, carregar pesos, varrer, entre outras. A atividade física no lazer abrangem os exercícios e os
Possíveis efeitos da pandemia de CoViD-19 sobre a prática de atividade física

Face ao cenário ora examinado, é fácil depreender que há diversos fatores que podem causar de diminuição à cessação da prática de atividade física: fatores psicossociais e fatores físicos. Dentre os fatores psicossociais estão os relativos ao estresse causado pelo fator traumático da pandemia, cuja mortalidade, em poucos meses, foi bastante alta levando à uma grande preocupação do indivíduo com sua própria saúde e com a saúde de seus entes queridos, além de serem agravados pelos fatores físicos de restrição social e da obrigatoriedade do uso de máscaras. Este último, promove desconforto, o que pode representar vários obstáculos extras para a motivação do indivíduo.

Essa situação somada às restrições quanto aos horários disponíveis nas academias de ginástica e de circulação em espaços públicos, adequados, podem ter dificultado e desencorajado a prática de atividade física em todo o tipo de população.

Problemas de segurança ambiental (iluminação, segurança pública, qualidade do piso, área apropriada), qualidade de transporte(79), estresse psicológico e saúde mental(69-71) per si são fatores determinantes da atividade física ${ }^{6}$, estando relacionados a níveis menores de prática.

É importante destacar que a relação entre nível de atividade física e saúde mental é bidirecional, isto significa que tanto maiores níveis de atividade física associam-se a menos sintomas em saúde mental(83) e mais sintomas em saúde mental levam a menores níveis de atividade física(69-71), o que sugere que, para quebrar a inércia a fim de romper o ciclo vicioso, é preciso maior esforço por parte do indivíduo. A literatura mostra que, tendo vencido a inércia e conseguido engajar-se na

esportesatividade física ocupacional, atividade física no lazer e atividade física em locomoção(81). Exercício: É uma subcategoria de atividade física que é planejada, estruturada, repetitiva e tem por objetivo melhorar ou manuter um ou mais componentes da aptidão física(82) Esporte pode ser definido como atividade física estruturada que é realizada segundo regras específicas, que demanda treinamento técnico e, geralmente, possui entre os objetivos a competição. 
prática de atividade física os benefícios à saúde mental são evidentes $(\mathbf{8 4 , 8 5})$.

\section{Benefícios da atividade física à saúde}

Quando se fala em benefícios à saúde promovidos pela prática de atividade física, há um quadro correlacional de vários aspectos da vida humana interligados. Por exemplo, a atividade física previne e trata, como coadjuvante, sintomas de ansiedade e depressão(85). A saúde mental por sua vez, relaciona-se ao estado do sistema imunológico(86-88). De acordo com a literatura, os mecanismos psicológicos relacionados à prática de exercícios favorecem os estados de humor, que podem ser decorrentes da distração e/ou da autoeficácia(84,86) e, ainda, reduz a inflamação por meio de vários processos fisiológicos diferentes $(\mathbf{8 6})$ promovendo melhora da função endotelial(89) e protegendo o sistema cardiocirculatório $(\mathbf{9 0 , 9 1 )}$ bem como melhora o funcionamento do cérebro e a cognição(92). E tem sido recomendada por cientistas para melhorar a saúde das populações, bem como o sistema imunológico, face à pandemia de CoViD-19(93,94).

\section{Conclusão}

O objetivo do presente estudo foi examinar o desenrolar histórico desse agravo à saúde, a CoViD-19, que afetou todas as vidas no planeta e, consequentemente, a produtividade em diversas áreas da vida humana. Procurouse também identificar os possíveis efeitos sobre a prática de atividade física.

A literatura é vasta e consistente quanto aos benefícios da atividade física para a saúde, o que leva à necessidade de estar sempre presente nas considerações dos gestores em saúde.

Estudos futuros devem examinar os efeitos sobre os hábitos saudáveis durante e após a pandemia, avaliando o impacto da diminuição dos níveis de atividade física na população em geral.

\section{Declaração de conflito de interesses}

Não há nenhum conflito de interesses em relação ao presente estudo.

\section{Declaração de financiamento}

Estudo conduzido sem financiamento.

\section{Referências}

1. Khan N. New Virus Discovered by Chinese Scientists Investigating Pneumonia Outbreak. Wall Street Journal. [Online] 2020; Available from: https://www.wsj.com/articles/new-virusdiscovered-by-chinese-scientistsinvestigating-pneumonia-outbreak11578485668 [Accessed: 16th May 2020]

2. Medronho RA, Bloch KV, Luiz RR, Werneck GL. Epidemiologia.. 2 ${ }^{\mathrm{a}}$ edição. São Paulo: Editora Atheneu; 2008.

3. CNN YX and NG. This Chinese doctor tried to save lives, but was silenced. Now he has coronavirus. [Online] CNN. Available from:

https://www.cnn.com/2020/02/03/asia/coro navirus-doctor-whistle-blower-intlhnk/index.html [Accessed: 7th December 2020]

4. WHO. Statement on the meeting of the International Health Regulations (2005) Emergency Committee regarding the outbreak of novel coronavirus 2019 (n$\mathrm{CoV}$ ) on 23 January 2020. [Online] World Health Organization. Available from: https://www.who.int/news/item/23-012020-statement-on-the-meeting-of-theinternational-health-regulations-(2005)emergency-committee-regarding-theoutbreak-of-novel-coronavirus-(2019ncov) [Accessed: 7th December 2020]

5. La Rosa G, Fratini M, Della Libera S, Iaconelli M, Muscillo M. Viral infections acquired indoors through airborne, droplet or contact transmission. Annali dell'Istituto Superiore Di Sanita. [Online] 2013;49(2): 124-132. Available from: doi:10.4415/ANN_13_02_03

6. Wang C, Horby PW, Hayden FG, Gao GF. A novel coronavirus outbreak of global health concern. The Lancet. [Online] Elsevier; 2020;395(10223): 470-473. Available from: doi:10.1016/S01406736(20)30185-9

7. de Wit E, van Doremalen N, Falzarano D, Munster VJ. SARS and MERS: recent insights into emerging coronaviruses. 
Nature Reviews. Microbiology. [Online] 2016;14(8): 523-534. Available from: doi:10.1038/nrmicro.2016.81

8. Coronavirus Update (Live): 67,512,016 Cases and 1,543,921 Deaths from COVID19 Virus Pandemic - Worldometer. [Online] Available from: https://www.worldometers.info/coronaviru s/ [Accessed: 7th December 2020]

9. Tang S, Mao Y, Jones RM, Tan Q, Ji JS, Li $\mathrm{N}$, et al. Aerosol transmission of SARSCoV-2? Evidence, prevention and control. Environment International. [Online] 2020;144: 106039. Available from: doi:10.1016/j.envint.2020.106039

10. CDC. Coronavirus Disease 2019 (COVID19). [Online] Centers for Disease Control and Prevention. Available from: https://www.cdc.gov/coronavirus/2019ncov/more/scientific-brief-sars-cov-2.html [Accessed: 7th December 2020]

11. Azuma K, Yanagi U, Kagi N, Kim H, Ogata M, Hayashi M. Environmental factors involved in SARS-CoV-2 transmission: effect and role of indoor environmental quality in the strategy for COVID-19 infection control. Environmental Health and Preventive Medicine. [Online] 2020;25(1): 66. Available from: doi:10.1186/s12199-020-00904-2

12. Wilson NM, Norton A, Young FP, Collins DW. Airborne transmission of severe acute respiratory syndrome coronavirus-2 to healthcare workers: a narrative review. Anaesthesia. [Online] 2020;75(8): 10861095. Available from: doi:10.1111/anae.15093

13. Stadnytskyi V, Bax CE, Bax A, Anfinrud P. The airborne lifetime of small speech droplets and their potential importance in SARS-CoV-2 transmission. Proceedings of the National Academy of Sciences. [Online] National Academy of Sciences; 2020;117(22): 11875-11877. Available from: doi:10.1073/pnas.2006874117

14. Pomés A, Chapman MD, Wünschmann S. Indoor Allergens and Allergic Respiratory
Disease. Current Allergy and Asthma Reports. [Online] 2016;16(6): 43. Available from: doi:10.1007/s11882-016-0622-9

15. Wang J, Engvall K, Smedje G, Nilsson H, Norbäck D. Current wheeze, asthma, respiratory infections, and rhinitis among adults in relation to inspection data and indoor measurements in single-family houses in Sweden-The BETSI study. Indoor Air. [Online] 2017;27(4): 725-736. Available from: doi:10.1111/ina.12363

16. Folha de São Paulo. Governo federal decreta estado de emergência para conter coronavirus no Brasil. [Online] Folha de S.Paulo. Available from: https://www1.folha.uol.com.br/equilibrioes aude/2020/02/governo-decreta-estado-deemergencia-por-causa-de-surto-docoronavirus.shtml [Accessed: 7th December 2020]

17. Terra. Confira as fotos dos desfiles do grupo especial em SP. [Online] Terra.com.br. Available from: https://www.terra.com.br/diversao/carnaval /sao-paulo/confira-as-fotos-dos-desfilesdo-grupo-especial-emsp,ad7ef53d77a5c2562c99fe91f920bc05rn 1ojdmi.html [Accessed: 7th December 2020]

18. UOL. Resultado do Carnaval 2020 - Rio de Janeiro. [Online] UOL. Available from: https://setor1.band.uol.com.br/resultadoclassificacao-carnaval-2020-rio/ [Accessed: 7th December 2020]

19. Riou J, Althaus CL. Pattern of early humanto-human transmission of Wuhan 2019 novel coronavirus (2019-nCoV), December 2019 to January 2020. Euro Surveillance: Bulletin Europeen Sur Les Maladies Transmissibles $=$ European Communicable Disease Bulletin. [Online] 2020;25(4). Available from: doi:10.2807/15607917.ES.2020.25.4.2000058

20. Bastos LFCS, https://www.facebook.com/pahowho. OPAS/OMS Brasil - Brasil confirma primeiro caso de infecção pelo novo coronavirus | OPAS/OMS. [Online] Pan 
American Health Organization / World Health Organization. Available from: https://www.paho.org/bra/index.php?optio $\mathrm{n}=\mathrm{com} \_$content\&view $=$article\&id $=6113: \mathrm{br}$ asil-confirma-primeiro-caso-de-infeccaopelo-novo-coronavirus \&Itemid $=812$ [Accessed: 7th December 2020]

21. WHO. A Joint Statement on Tourism and COVID-19 - UNWTO and WHO Call for Responsibility and Coordination. [Online] Available from: https://www.who.int/news/item/27-022020-a-joint-statement-on-tourism-andcovid-19---unwto-and-who-call-forresponsibility-and-coordination [Accessed: 7th December 2020]

22. Gautret P, Lagier J-C, Parola P, Hoang VT, Meddeb L, Sevestre J, et al. Clinical and microbiological effect of a combination of hydroxychloroquine and azithromycin in 80 COVID-19 patients with at least a six-day follow up: A pilot observational study. Travel Medicine and Infectious Disease. [Online] 2020;34: 101663. Available from: doi:10.1016/j.tmaid.2020.101663

23. Liu J, Cao R, Xu M, Wang X, Zhang H, Hu $\mathrm{H}$, et al. Hydroxychloroquine, a less toxic derivative of chloroquine, is effective in inhibiting SARS-CoV-2 infection in vitro. Cell Discovery. [Online] Nature Publishing Group; 2020;6(1): 1-4. Available from: doi:10.1038/s41421-020-0156-0

24. WHO. WHO statement on cases of COVID19 surpassing 100 000. [Online] Available from: https://www.who.int/news/item/0703-2020-who-statement-on-cases-of-covid19-surpassing-100-000 [Accessed: 7th December 2020]

25. Worldometers. Coronavirus Update (Live): 69,441,054 Cases and 1,579,397 Deaths from COVID-19 Virus Pandemic Worldometer. [Online] Worldometers COVID-19 Coronavirus Pandemic. Available from: https://www.worldometers.info/coronaviru s/\#countries [Accessed: 10th December 2020]
26. Adam D. Special report: The simulations driving the world's response to COVID-19. Nature. [Online] Nature Publishing Group; 2020;580(7803): 316-318. Available from: doi:10.1038/d41586-020-01003-6

27. Oliveira PI. Agência Brasil explica: entenda o que é o lockdown. [Online] Agência Brasil. Available from: https://agenciabrasil.ebc.com.br/geral/notic $\mathrm{ia} / 2020-05 /$ agencia-brasil-explica-entendao-que-e-o-lockdown [Accessed: 7th December 2020]

28. O Globo. Fome ameaça matar mais do que a Covid-19 em 2020, indicam projeções. [Online] Economia. Available from: https://economia.ig.com.br/2020-0831/fome-ameaca-matar-mais-do-que-acovid-19-em-2020-indicam-projecoes.html [Accessed: 7th December 2020]

29. Hare DL, Toukhsati SR, Johansson P, Jaarsma T. Depression and cardiovascular disease: a clinical review. European Heart Journal. [Online] 2014;35(21): 1365-1372. Available doi:10.1093/eurheartj/eht462

30. Wang J, Lloyd-Evans B, Giacco D, Forsyth R, Nebo C, Mann F, et al. Social isolation in mental health: a conceptual and methodological review. Social Psychiatry and Psychiatric Epidemiology. [Online] 2017;52(12): 1451-1461. Available from: doi:10.1007/s00127-017-1446-1

31. Kobayashi LC, Steptoe A. Social Isolation, Loneliness, and Health Behaviors at Older Ages: Longitudinal Cohort Study. Annals of Behavioral Medicine: A Publication of the Society of Behavioral Medicine. [Online] 2018;52(7): 582-593. Available from: doi:10.1093/abm/kax033

32. Schrempft S, Jackowska M, Hamer M, Steptoe A. Associations between social isolation, loneliness, and objective physical activity in older men and women. $B M C$ public health. [Online] 2019;19(1): 74. Available from: doi:10.1186/s12889-0196424-y 
33. WHO. ICC-WHO Joint Statement: An unprecedented private sector call to action to tackle COVID-19. [Online] Available from: https://www.who.int/news/item/1603-2020-icc-who-joint-statement-anunprecedented-private-sector-call-toaction-to-tackle-covid-19 [Accessed: 7th December 2020]

34. GOV.BR. Entra em vigor estado de calamidade pública no Brasil. [Online] Planalto. Available from: https://www.gov.br/planalto/ptbr/acompanhe-oplanalto/noticias/2020/03/entra-em-vigorestado-de-calamidade-publica-no-brasil [Accessed: 7th December 2020]

35. WHO. Joint Statement by QU Dongyu, Tedros Adhanom Ghebreyesus and Roberto Azevedo, Directors-General of the Food and Agriculture Organization of the United Nations (FAO), the World Health Organization (WHO) and the World Trade Organization (WTO). [Online] Available from: https://www.who.int/news/item/3003-2020-joint-statement-by-qu-dongyutedros-adhanom-ghebreyesus-and-robertoazevedo-directors-general-of-the-food-andagriculture-organization-of-the-unitednations-(fao)-the-world-healthorganization-(who)-and-the-world-tradeorganization-(wto) [Accessed: 7th December 2020]

36. WHO. Statement of the Twenty-Fourth IHR Emergency Committee. [Online] Available from: https://www.who.int/news/item/0804-2020-statement-of-the-twenty-fourthihr-emergency-committee [Accessed: 7th December 2020]

37. WHO. Joint Leaders' statement - Violence against children: $A$ hidden crisis of the COVID-19 pandemic. [Online] Available from: https://www.who.int/news/item/0804-2020-joint-leader-s-statement--violence-against-children-a-hidden-crisisof-the-covid-19-pandemic [Accessed: 7th December 2020]

38. WHO. Public statement for collaboration on COVID-19 vaccine development. [Online] Available from: https://www.who.int/news/item/13-042020-public-statement-for-collaborationon-covid-19-vaccine-development [Accessed: 7th December 2020]

39. UOL. STF dá poder a estados para atuar contra covid-19 e impõe revés a Bolsonaro. [Online] Available from: https://noticias.uol.com.br/saude/ultimasnoticias/redacao/2020/04/15/stf-tem-4votos-a-favor-de-autonomia-degovernadores-durante-a-pandemia.htm [Accessed: 7th December 2020]

40. G1. Mandetta anuncia em rede social que foi demitido por Bolsonaro do Ministério da Saúde. [Online] G1. Available from: https://g1.globo.com/politica/noticia/2020/ 04/16/mandetta-anuncia-em-rede-socialque-foi-demitido-do-ministerio-dasaude.ghtml [Accessed: 7th December 2020]

41. Gautret P, Lagier J-C, Parola P, Hoang VT, Meddeb L, Mailhe $\mathrm{M}$, et al. Hydroxychloroquine and azithromycin as a treatment of COVID-19: results of an openlabel non-randomized clinical trial. [Online] Infectious Diseases (except HIV/AIDS), 2020 Mar [Accessed: 7th December 2020]. Available from: doi:10.1101/2020.03.16.20037135 [Accessed: 7th December 2020]

42. Colson P, Rolain J-M, Lagier J-C, Brouqui $\mathrm{P}$, Raoult D. Chloroquine and hydroxychloroquine as available weapons to fight COVID-19. International Journal of Antimicrobial Agents. [Online] 2020;55(4): 105932. Available from: doi:10.1016/j.ijantimicag.2020.105932

43. Heidary F, Gharebaghi R. Ivermectin: a systematic review from antiviral effects to COVID-19 complementary regimen. The Journal of Antibiotics. [Online] Nature Publishing Group; 2020;73(9): 593-602. Available from: doi:10.1038/s41429-0200336-z

44. Alexander J, Tinkov A, Strand TA, Alehagen U, Skalny A, Aaseth J. Early Nutritional Interventions with Zinc, Selenium and Vitamin D for Raising Anti- 
Viral Resistance Against Progressive COVID-19. Nutrients. [Online] 2020;12(8). Available from: doi:10.3390/nu12082358

45. Freitas TIS, editor. Protocolo para atendimento da CoViD-19 na atenção primária e hospitalar. Editora da Universidade Federal do Piauí - EDUFPI. $2020 ; 41$.

46. Jornal Aliança. Porto Feliz dá exemplo de como vencer o Covid-19 | Jornal Aliança. [Online] Jornal Aliança. Available from: https://jornalalianca.com.br/2020/05/29/po rto-feliz-da-exemplo-de-como-vencer-ocovid-19/ [Accessed: 7th December 2020]

47. Brasil, Ministério da Saúde. Orientações do Ministério da Saúde para manuseio medicamentoso precoce de pacientes com diagnóstico da CoViD-19 - Nota informativa no. 17/2020 - SE/GAB/SE/MS. Ministério da Saúde; 2020.

48. IBGE. Porto Feliz (SP)|Cidades e Estados. [Online] Instituto Brasileiro de Geografia e Estatística. Available from: https://www.ibge.gov.br/cidades-eestados/sp/porto-feliz.html [Accessed: 17th December 2020]

49. Rothman K, Greenland S, Lash T. Epidemiologia Moderna - $3^{a}$ Edição. Porto Alegre, RS: Artmed Editora; 2016. 887 p.

50. GOV.BR. Serviços essenciais: decreto presidencial inclui mais atividades à lista do que não podem parar durante a pandemia. [Online] Casa Civil. Available from: https://www.gov.br/casacivil/ptbr/assuntos/noticias/2020/maio/servicosessenciais-decreto-presidencial-incluimais-atividades-a-lista-do-que-nao-podemparar-durante-a-pandemia [Accessed: 7th December 2020]

51. O Globo. Coronavirus: ONU alerta que crise economica pode matar centenas de milhares de crianças em 2020. [Online] O Globo. Available from: https://oglobo.globo.com/mundo/coronavir us-onu-alerta-que-crise-economica-podematar-centenas-de-milhares-de-criancas- em-2020-1-24375960 [Accessed: 9th December 2020]

52. Torales J, O’Higgins M, Castaldelli-Maia JM, Ventriglio A. The outbreak of COVID19 coronavirus and its impact on global mental health. The International Journal of Social Psychiatry. [Online] 2020;66(4): 317-320. Available from: doi:10.1177/0020764020915212

53. Talevi D, Socci V, Carai M, Carnaghi G, Faleri S, Trebbi E, et al. Mental health outcomes of the CoViD-19 pandemic. Rivista Di Psichiatria. [Online] 2020;55(3): 137-144. Available from: doi:10.1708/3382.33569

54. Pfefferbaum B, North CS. Mental Health and the Covid-19 Pandemic. The New England Journal of Medicine. [Online] 2020;383(6): 510-512. Available from: doi:10.1056/NEJMp2008017

55. Shan Y, Wu W, Fan W, Haahtela T, Zhang G. House dust microbiome and human health risks. International Microbiology: The Official Journal of the Spanish Society for Microbiology. [Online] 2019;22(3): 297-304. Available from: doi:10.1007/s10123-019-00057-5

56. Kechichian E, Ezzedine K. Vitamin D and the Skin: An Update for Dermatologists. American Journal of Clinical Dermatology. [Online] 2018;19(2): 223-235. Available from: doi:10.1007/s40257-017-0323-8

57. Matsushima Y, Mizutani K, Yamaguchi Y, Yamanaka K. Vitamin D is no substitute for the sun. The Journal of Allergy and Clinical Immunology. [Online] 2019;143(3): 929931. Available from: doi:10.1016/j.jaci.2019.01.004

58. Prietl B, Treiber G, Pieber TR, Amrein K. Vitamin D and immune function. Nutrients. [Online] 2013;5(7): 2502-2521. Available from: doi:10.3390/nu5072502

59. FIOCRUZ-BA. É \#FAKE que alto nível de vitamina $D$ no organismo reduza a quase zero a chance de morte pela Covid-19 Coronavírus. [Online] 2020. Available 
from:

https://coronavirus.bahia.fiocruz.br/e-fakeque-alto-nivel-de-vitamina-d-noorganismo-reduza-a-quase-zero-a-chancede-morte-pela-covid-19/ [Accessed: 9th December 2020]

60. Catraca Livre. Suplementação de vitamina D não protege contra coronavirus e pode trazer riscos. [Online] Catraca Livre. Available from: https://catracalivre.com.br/saude-bemestar/suplementacao-de-vitamina-d-naoprotege-contra-coronavirus-e-pode-trazerriscos/ [Accessed: 9th December 2020]

61. Prefeitura do Rio. Rio Contra o Corona. [Online] Prefeitura do Rio de Janeiro; 2020. Available from: https://www.rio.rj.gov.br/documents/73801 /fe29064a-99f7-45b4-8d8f-c1547f201729

[Accessed: 9th December 2020]

62. Hewison M. An update on vitamin D and human immunity. Clinical Endocrinology. [Online] 2012;76(3): 315-325. Available from: doi:10.1111/j.13652265.2011.04261.x

63. Hernández JL, Nan D, Fernandez-Ayala M, García-Unzueta M, Hernández-Hernández MA, López-Hoyos M, et al. Vitamin D Status in Hospitalized Patients with SARSCoV-2 Infection. The Journal of Clinical Endocrinology \& Metabolism. [Online] 2020;(dgaa733). Available from: doi:10.1210/clinem/dgaa733 [Accessed: 9th December 2020]

64. Grant WB, Lahore H, McDonnell SL, Baggerly CA, French CB, Aliano JL, et al. Evidence that Vitamin D Supplementation Could Reduce Risk of Influenza and COVID-19 Infections and Deaths. Nutrients. [Online] Multidisciplinary Digital Publishing Institute; 2020;12(4): 988. Available from: doi:10.3390/nu12040988

65. Kato TA, Sartorius N, Shinfuku N. Forced social isolation due to COVID-19 and consequent mental health problems: Lessons from hikikomori. Psychiatry and Clinical Neurosciences. [Online] 2020;
Available from: doi:10.1111/pcn.13112 [Accessed: 9th December 2020]

66. Mari J de J, Oquendo MA, Mari J de J, Oquendo MA. Mental health consequences of COVID-19: the next global pandemic. Trends in Psychiatry and Psychotherapy. [Online] Associação de Psiquiatria do Rio Grande do Sul; 2020;42(3): 219-220. Available from: doi:10.1590/2237-60892020-0081

67. Reiche EMV, Nunes SOV, Morimoto HK. Stress, depression, the immune system, and cancer. The Lancet. Oncology. [Online] 2004;5(10): 617-625. Available from: doi:10.1016/S1470-2045(04)01597-9

68. Kaye J, Morton J, Bowcutt M, Maupin D. Stress, Depression, and Psychoneuroimmunology. Journal of Neuroscience Nursing. 2000;32(2): 93100.

69. Delgado-Floody P, Guzmán-Guzmán IP, Caamaño-Navarrete F, Jerez-Mayorga D, Zulic-Agramunt C, Cofré-Lizama A. Depression is associated with lower levels of physical activity, body image dissatisfaction, and obesity in Chilean preadolescents. Psychology, Health \& Medicine. [Online] Taylor \& Francis; 2020;0(0): 1-14. Available from: doi:10.1080/13548506.2020.1817958

70. Minaeva O, Booij SH, Lamers F, Antypa N, Schoevers RA, Wichers $M$, et al. Level and timing of physical activity during normal daily life in depressed and non-depressed individuals. Translational Psychiatry. [Online] Nature Publishing Group; 2020;10(1): 1-11. Available from: doi:10.1038/s41398-020-00952-w

71. Martins LCX, Lopes CS. Rank, job stress, psychological distress and physical activity among military personnel. BMC Public Health. [Online] 2013;13: 716. Available from: doi:10.1186/1471-2458-13-716

72. WHO. Mask use in the context of COVID19: interim guidance, 1 December 2020. Worl Health Organization. World Health Organization; 2020; 1-22. 
73. Fikenzer S, Uhe T, Lavall D, Rudolph U, Falz R, Busse M, et al. Effects of surgical and FFP2/N95 face masks on cardiopulmonary exercise capacity. Clinical Research in Cardiology. [Online] 2020; 1-9. Available from: doi:10.1007/s00392-020-01704-y

74. Lee HP, Wang DY. Objective assessment of increase in breathing resistance of $\mathrm{N} 95$ respirators on human subjects. The Annals of Occupational Hygiene. [Online] 2011;55(8): 917-921. Available from: doi:10.1093/annhyg/mer065

75. Editora Melhoramentos. Dicionário de Português Michaelis. 2020. Available from: https://michaelis.uol.com.br/modernoportugues/busca/portuguesbrasileiro/holismo/ [Accessed: 16th October 2020]

76. Person E, Lemercier C, Royer A, Reychler G. Effet du port d'un masque de soins lors d'un test de marche de six minutes chez des sujets sains. Revue des Maladies Respiratoires. [Online] 2018;35(3): 264268. Available from: doi:10.1016/j.rmr.2017.01.010

77. Harber P, Santiago S, Bansal S, Liu Y, Yun $\mathrm{D}, \mathrm{Wu} \mathrm{S}$. Respirator physiologic impact in persons with mild respiratory disease. Journal of Occupational and Environmental Medicine. [Online] 2010;52(2): 155-162. Available from: doi:10.1097/JOM.0b013e3181ca0ec9

78. SBME. Informes e Notas da SBMEE sobre Coronavírus e Exercício Físico. [Online] Sociedade Brasileira de Medicina do Exercício e do Esporte. 2020. Available from:

http://www.medicinadoesporte.org.br/infor mes-da-sbmee-sobre-coronavirus-eexercicio-fisico/ [Accessed: 17th December 2020]

79. Carlin A, Perchoux C, Puggina A, Aleksovska K, Buck C, Burns C, et al. A life course examination of the physical environmental determinants of physical activity behaviour: A 'Determinants of Diet and Physical Activity' (DEDIPAC) umbrella systematic literature review. PloS One. [Online] 2017;12(8): e0182083. Available from: doi:10.1371/journal.pone.0182083

80. Caspersen CJ, Powell KE, Christenson GM. Physical activity, exercise, and physical fitness: definitions and distinctions for health-related research. Public Health Reports. 1985;100(2): 126-131.

81. Baecke JA, Burema J, Frijters JE. A short questionnaire for the measurement of habitual physical activity in epidemiological studies. The American Journal of Clinical Nutrition. [Online] 1982;36(5): 936-942. Available from: doi:10.1093/ajen/36.5.936

82. U.S. Department of Health and Human Services. Physical Activity Guidelines for Americans. [Online] U.S. Department of Health and Human Services; 2008. Available from: https://health.gov/sites/default/files/201909/paguide.pdf [Accessed: 9th December 2020]

83. De Mello MT, Lemos V de A, Antunes HKM, Bittencourt L, Santos-Silva R, Tufik S. Relationship between physical activity and depression and anxiety symptoms: A population study. Journal of Affective Disorders. [Online] 2013;149(1): 241-246. Available from: doi:10.1016/j.jad.2013.01.035

84. Peluso MAM, Andrade LHSG de. Physical activity and mental health: the association between exercise and mood. Clinics. [Online] 2005;60(1): 61-70. Available from: doi:10.1590/S180759322005000100012

85. Hamer M, Stamatakis E, Steptoe A. Doseresponse relationship between physical activity and mental health: the Scottish Health Survey. British Journal of Sports Medicine. [Online] 2009;43(14): 11111114. Available from: doi:10.1136/bjsm.2008.046243

86. Mikkelsen K, Stojanovska L, Polenakovic M, Bosevski M, Apostolopoulos V. 
Exercise and mental health. Maturitas. [Online] 2017;106: 48-56. Available from: doi:10.1016/j.maturitas.2017.09.003

87. Salim S, Chugh G, Asghar M. Inflammation in anxiety. Advances in Protein Chemistry and Structural Biology. [Online] 2012;88: 1-25. Available from: doi:10.1016/B978-012-398314-5.00001-5

88. Dantzer R, O'Connor JC, Freund GG, Johnson RW, Kelley KW. From inflammation to sickness and depression: when the immune system subjugates the brain. Nature Reviews. Neuroscience. [Online] 2008;9(1): 46-56. Available from: doi:10.1038/nrn2297

89. Green DJ, Maiorana A, O’Driscoll G, Taylor R. Effect of exercise training on endothelium-derived nitric oxide function in humans. The Journal of Physiology. [Online] 2004;561(Pt 1): 1-25. Available from: doi:10.1113/jphysiol.2004.068197

90. Lavie CJ, Arena R, Swift DL, Johannsen NM, Sui X, Lee D, et al. Exercise and the Cardiovascular System: Clinical Science and Cardiovascular Outcomes. Circulation research. [Online] 2015;117(2): 207-219. Available from: doi:10.1161/CIRCRESAHA.117.305205

91. Mortensen SP, Nyberg M, Gliemann L, Thaning P, Saltin B, Hellsten Y. Exercise training modulates functional sympatholysis and $\alpha$-adrenergic vasoconstrictor responsiveness in hypertensive and normotensive individuals. The Journal of Physiology. [Online] 2014;592(Pt 14): 3063-3073. Available from: doi:10.1113/jphysiol.2014.273722

92. Rêgo ML, Cabral DA, Costa EC, Fontes EB. Physical Exercise for Individuals with Hypertension: It Is Time to Emphasize its Benefits on the Brain and Cognition. Clinical Medicine Insights. Cardiology. [Online] 2019;13. Available from: doi:10.1177/1179546819839411

[Accessed: 10th December 2020]

93. Woods JA, Hutchinson NT, Powers SK, Roberts WO, Gomez-Cabrera MC, Radak
Z, et al. The COVID-19 pandemic and physical activity. Sports Medicine and Health Science. [Online] 2020;2(2): 55-64. Available from: doi:10.1016/j.smhs.2020.05.006

94. da Silveira MP, da Silva Fagundes KK, Bizuti MR, Starck É, Rossi RC, de Resende e Silva DT. Physical exercise as a tool to help the immune system against COVID-19: an integrative review of the current literature. Clinical and Experimental Medicine. [Online] 2020; Available from: doi:10.1007/s10238020-00650-3 [Accessed: 10th December 2020] 